\title{
Les codes d'éthique des entreprises manufacturières et leurs mécanismes de renforcement interne
}

\author{
Michel DION ${ }^{1}$ et Louis MARQUIS² \\ Université de Sherbrooke
}

\section{Introduction}

Les codes d'éthique d'entreprises sont apparus peu après le scandale du Watergate aux États-Unis, même si quelques entreprises s'étaient déjà dotées de documents similaires dans le passé. Le scandale en question, bien que politique, avait créé tout un émoi dans la société américaine et avait suscité, en peu de temps, une perte de confiance de la population envers ses grands leaders, qu'ils soient issus des milieux économiques comme des milieux d'affaires. Quelques grands scandales dans le monde des affaires ont aussi attisé la flamme de cette perte de confiance envers les dirigeants de grandes entreprises. Les cas de la Ford Pinto (sécurité des produits), de Lockheed au Japon (pots-de-vin) et de Goodrich et l'avion militaire A\&D (falsification des tests de laboratoire), tous survenus au début des années 1970, ont été très marquants et ont peu à peu «creusé la tombe» de la confiance du public envers les dirigeants de grandes entreprises. D'autres scandales tout aussi retentissants sont survenus à la fin des années 1970 et au début des années 1980, tel Union Carbide et le désastre de Bhopal. Ces scandales et bien d'autres ont tôt fait, aux États-Unis, de miner la confiance des consommateurs envers les grandes entreprises américaines («seconde grande vague des codes d'éthique »: milieu des années 1980). Il faut dire que la Commission Treadway avait, en 1987, invité les compagnies américaines à se doter de codes d'éthique pour éviter les fraudes $^{3}$. Les entreprises ont donc progressivement réagi, en voulant sauver cette confiance si précieuse par laquelle l'entreprise s'assure la fidélité de ses consommateurs, sa part de marché et donc sa viabilité à long terme, en essayant de démontrer comment elles pouvaient s'auto-réglementer de façon à prévenir de telles crises, et si par contre elles devaient survenir, à les gérer de manière socialement responsable. Les grandes entreprises américaines, qui ont initié les deux premières grandes vagues de codes d'éthique, étaient poussées en cela par l'opinion publique américaine scandalisée qui demandait à l'État d'intervenir en réglementant davantage le milieu des affaires. Réaction vive des entreprises à cette menace de réglementation additionnelle : les entreprises voyaient cette réglementation comme un fardeau additionnel qui ne pourrait que réduire leur compétitivité. D'où l'idée du code d'éthique d'entreprise, qui permet d'éviter la réglementation gouvernementale, de satisfaire les attentes du public et ainsi de s'assurer d'une bonne image corporative et de la fidélité de ses clients. La «troisième vague des codes d'éthique»a été l'effet de scandales d'entreprises américaines ayant une image de bon citoyen corporatif dans l'opinion publique. Ce fut le cas de Levi Strauss qui, en 1992, a dû se doter de normes de protection de l'environnement, de santé et sécurité au travail et de normes de travail (salaires et avantages sociaux, travail des enfants, travail forcé, non-discrimination). La même année, Sears s'est aussi dotée de normes de conduite afin d'interdire le travail forcé ou l'embauche de détenus pour la fabrication de produits destinés à la Chine. Reebok a fait de même par la suite. Dans bien des cas malheureusement, ces codes ne furent pas appliqués à la lettre ${ }^{4}$.

Quelques études existent déjà sur les codes d'éthique d'entreprises au Canada, mais bien peu par rapport à la masse de documentation disponible dans le cas des compagnies américaines. Dans cet article, nous partirons ainsi d'une analyse de contenu de codes d'éthique d'entreprises manufacturières, ce qui a été 
très peu l'objet de recherches spécifiques, même aux États-Unis. Cet article est basé sur une analyse de contenu de 35 codes d'éthique qui ont été reçus par les chercheurs dans le cadre d'une enquête provinciale réalisée en 1993 (225 répondants à l'enquête, 85 entreprises ayant des codes d'éthique), auprès des entreprises manufacturières de plus de cent employés faisant affaires au Québec. Dans un premier temps, nous présentons les résultats de notre étude du traitement de chacun des sujets couverts par les codes d'éthique, la présence des interdictions, tolérances et jugements. Dans une seconde section, nous poserons un regard critique sur les différences observées selon que ces entreprises sont québécoises, canadiennes, américaines ou européennes. Enfin, nous ferons voir combien la vision contractualiste et la vision communautariste peuvent donner lieu à deux éthiques d'entreprise bien différentes, pour ensuite conclure sur l'apport effectif de l'éthique des affaires.

\section{Analyse de contenu des codes d'éthique}

L'analyse de contenu des codes d'éthique d'entreprises manufacturières s'est ici attachée à quelques critères, les autres ayant fait l'objet d'une précédente publication $^{5}$ : le traitement relatif des différents conflits éthiques, les interdictions, tolérances et jugements qui les caractérisent, les mécanismes de dénonciation et de réponse aux questions d'interprétation du code d'éthique de l'entreprise. Il sera également fait état de l'affidavit (comme méthode de contrôle du comportement éthique) et du niveau de référence aux lois à l'intérieur des codes d'éthique d'entreprises.

\section{Le traitement des sujets}

Selon le tableau 1, les sources de conflits éthiques ont été énumérées à partir du nombre d'entreprises qui en ont traité dans leurs codes d'éthique. Cela nous donne déjà une bonne idée de l'importance de ces sujets pour les entreprises en question. Ainsi, la plupart des entreprises discutent des conflits d'intérêts $(77,1 \%)$, de la tenue des livres $(68,5 \%)$ et de l'utilisation de renseignements confidentiels $(60 \%)$. Plus de la moitié $(54,3 \%)$ traitent de la concurrence déloyale. Le quart des entreprises $(25,7 \%)$ ont couvert la protection de l'environnement et les relations avec les clients. Très peu de codes ont une section portant sur l'utilisation des biens corporatifs à des fins personnelles $(8,5 \%)$, sur l'alcoolisme et la toxicomanie en milieu de travail ou sur le harcèlement sexuel $(5,7 \%)$.
Nous pouvons également remarquer que le nombre de sujets couverts varie de façon inversement proportionnelle à la catégorie du nombre d'entreprises en question. Ainsi, entre les transactions d'initiés et la protection des biens corporatifs se retrouvent 11 sujets qui sont couverts par 2 à 5 entreprises. Dans la catégorie de 6 à 10 compagnies, nous retrouvons 8 sujets traités (entre les relations avec les clients et les activités politiques des employés). Dans la catégorie de 11 à 15 entreprises, trois sujets seulement sont traités, et on se rend ainsi jusqu'à la dernière catégorie (26 compagnies et plus), où il n'est question que des conflits d'intérêts. Par ailleurs, les sujets sur lesquels certains codes d'éthique s'étendent le plus longuement sont la concurrence déloyale (jusqu'à $65 \%$ ), l'utilisation de renseignements confidentiels et la responsabilité sociale de l'entreprise (50\%), les conflits d'intérêts (40\%), les paiements douteux et les cadeaux (35\%), les relations avec les employés et les gouvernements (30\%). Dans la majorité des cas, il s'agit de catégories réunissant entre 11 et 27 compagnies, donc les catégories elles-mêmes qui comportent le moins de sujets traités. Enfin, lorsque nous regardons de plus près l'étendue du traitement des différentes sources de conflits éthiques, certains recoupements peuvent être faits. Dans le cas de l'utilisation de renseignements confidentiels et de la responsabilité sociale de l'entreprise ${ }^{6}(0-50 \%)$, la majorité des entreprises accordent à ces sujets au plus $15 \%$ du texte de leurs codes d'éthique. En ce qui concerne les paiements douteux et les cadeaux (0-35\%), la majorité des compagnies n'accordent pas plus de $15 \%$ pour le traitement de ces sujets dans leurs codes d'éthique. En ce qui a trait aux relations avec les employés et avec les gouvernements (0-30\%), la majorité des entreprises leur accordent $15 \%$ et moins du texte de leur code d'éthique. Dans le cas des transactions d'initiés et de la santé et sécurité au travail (0-20\%), il n'y a pas de convergence des résultats. Dans le cas de la catégorie 0-15\%, aucune conclusion ne s'impose, alors que dans la catégorie 0-10\%, la majorité des entreprises n'attribuent pas plus de $5 \%$ du texte à chacun des 5 sujets en question, soit les relations avec le public, avec les actionnaires, avec les fournisseurs, les activités politiques des employés, l'équité en matière d'emploi.

Dans certains codes d'éthique d'entreprises, il n'y a aucun regroupement de sujets. Par ailleurs, dans ceux qui comportent de tels regroupements, nous avons répertorié sept modes de structuration de l'éthique de l'entreprise. 


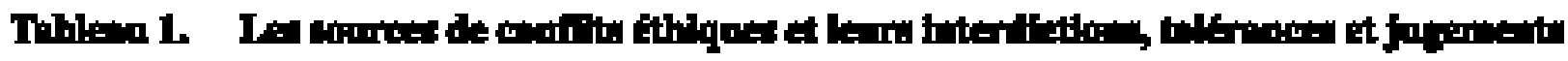

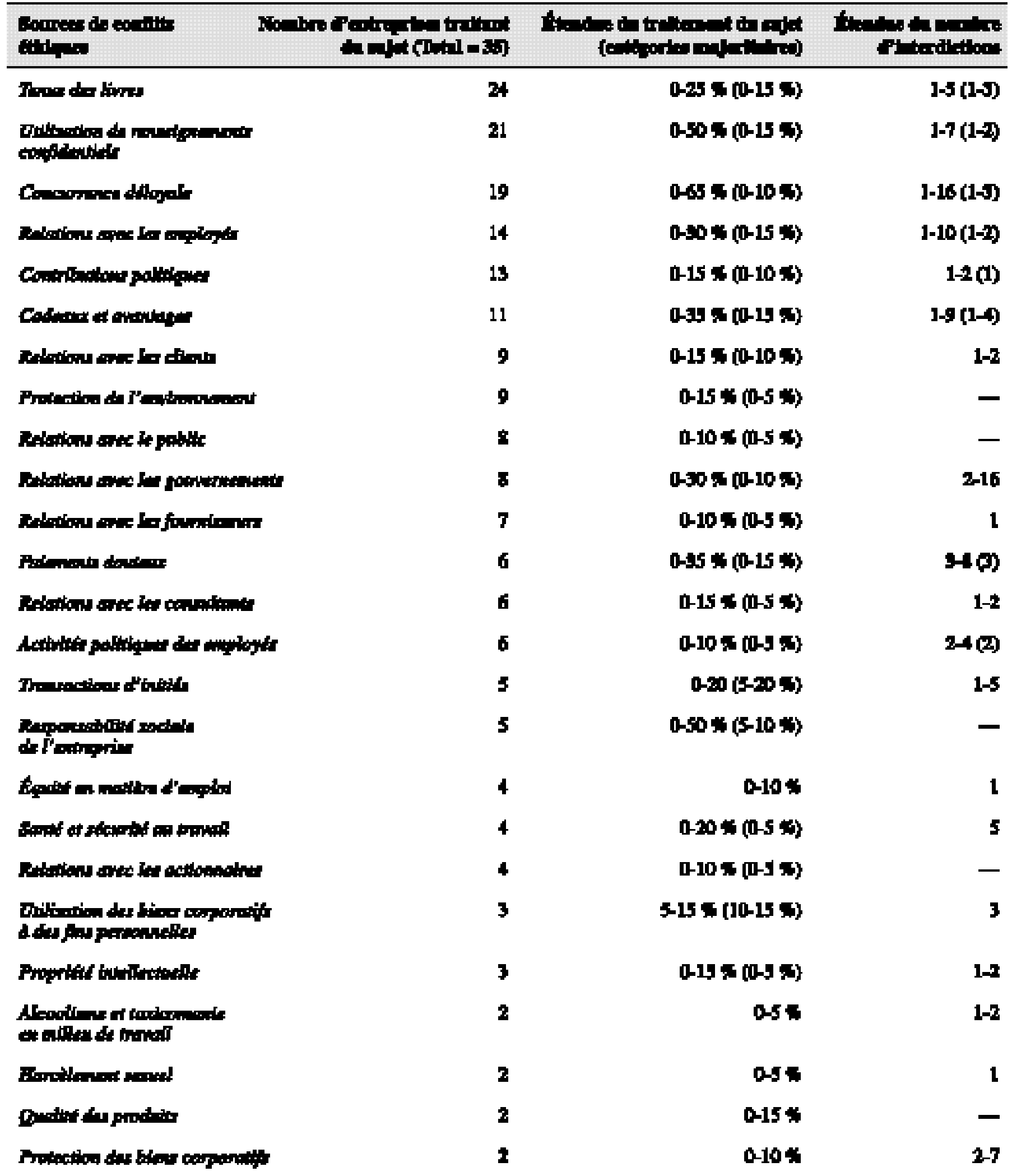




\section{Les quatre modes les plus courants}

\section{1) Regroupement des sujets par thématiques}

Nombre de sujets sont regroupés sous une rubrique générique "pratiques commerciales »: tenue des livres, protection des actifs corporatifs, divulgation de renseignements confidentiels, conflits d'intérêts, relations avec la communauté, propriété intellectuelle, activités politiques des employés. D'autres sujets sont souvent regroupés sous une rubrique dont les titres varient mais sont toujours très vagues (conduite au travail, éthique en milieu de travail, emploi et lieu de travail) : santé et sécurité au travail, protection de l'environnement, alcoolisme et toxicomanie en milieu de travail, divulgation de renseignements confidentiels, cadeaux, contributions politiques, utilisation des biens corporatifs à des fins personnelles, équité en matière d'emploi, harcèlement et discrimination, relations avec les consultants. Quant à la section touchant les conflits d'intérêts, son contenu varie grandement d'un code à l'autre. Peuvent être ainsi couverts par cette section les sujets suivants : participation financière des employés ou des membres de leur famille dans une entreprise concurrente, deuxième emploi, propriété intellectuelle, paiements douteux, acceptation, sollicitation et octroi de cadeaux, tenue des livres, transactions d'initiés, divulgation de renseignements confidentiels, utilisation des biens corporatifs à des fins personnelles, participation des employés dans des organismes communautaires ou de charité, activités politiques des employés. En ce qui concerne les relations avec les gouvernements, ce thème regroupe souvent l'engagement d'anciens politiciens ou administrateurs publics, les contributions politiques, les contrats gouvernementaux, le lobbying et les activités politiques des employés. Enfin, la santé et sécurité au travail, la protection de l'environnement et la qualité et sécurité des produits sont souvent regroupés dans la même section.

2) Regroupement par intervenants du milieu des affaires («stakeholders»)
Il s'agit généralement de la division suivante :

- Clients et fournisseurs

Conflits d'intérêts, concurrence déloyale, qualité et sécurité des produits, divulgation de renseignements confidentiels, propriété intellectuelle, publicité trompeuse et fausse représentation;

- Employés

Équité en matière d'emploi, alcoolisme et toxicomanie en milieu de travail, droit à la vie privée des employés;

- Actionnaires

Propriété intellectuelle, tenue des livres;

- Compétiteurs

Concurrence déloyale, publicité trompeuse et fausse représentation;

- Relations avec la communauté

Contributions politiques, activités politiques des employés, corruption, protection de l'environnement.

3) Regroupement des sujets par valeurs organi sationnelles

Les sujets sont ici regroupés selon chaque valeur organisationnelle d'importance. Il peut ainsi s'agir d'une division comme celle-ci :

- Équité

Non-discrimination, concurrence déloyale;

- Responsabilité corporative

Contributions politiques, responsabilité sociale;

- Confiance

Gonflement des comptes de dépenses, vol et fraude, équité en matière d'emploi, santé et sécurité au travail, violence au travail;

- Objectivité

Cadeaux, conflits d'intérêts;

- Intégrité 
Conflits d'intérêts, propriété intellectuelle, cadeaux, détournements de fonds, tenue des livres;

- Responsabilité individuelle Harcèlement;

- Loyauté

Utilisation des biens corporatifs à des fins personnelles;

- Respect

Discrimination et harcèlement, santé et sécurité au travail, protection de l'environnement.

4) Regroupement hybride des sujets par théma tiques, intervenants et valeurs organisation nelles

Le plus souvent, il s'agit d'un regroupement qui combine l'utilisation de thématiques et de catégories d'intervenants. Assez souvent, nous voyons aussi un regroupement combinant thématiques et valeurs organisationnelles.

\section{Les trois modes additionnels (moins courants)}

1) Regroupement des sujets par thématiques et par une liste de sujets non regroupés

Ces derniers sujets peuvent être parmi les suivants : conflits d'intérêts, divulgation de renseignements confidentiels, transaction d'initiés, second emploi, cadeaux, protection de l'environnement, santé et sécurité au travail, utilisation des biens corporatifs à des fins personnelles, propriété intellectuelle, activités politiques des employés;

2) Regroupement des sujets en distinguant la conduite à l'égard de la compagnie et la conduite à l'égard de tiers

La conduite à l'égard de la compagnie regroupe alors souvent les conflits d'intérêts (incluant les cadeaux, le deuxième emploi, la participation financière chez des concurrents), la divulgation de renseignements confidentiels (incluant les transactions d'initiés) et l'utilisation des biens corporatifs à des fins person- nelles (incluant les contributions politiques, les cadeaux et la propriété intellectuelle). La conduite à l'égard des tiers inclut alors les relations avec les compétiteurs (concurrence déloyale, divulgation de renseignements confidentiels), les relations avec les distributeurs et les fournisseurs (divulgation de renseignements confidentiels).

\section{3) Regroupement des sujets en distinguant «lois»} et «déontologie»

Le thème des lois couvre alors la concurrence déloyale, la discrimination, les transactions d'initiés, la propriété intellectuelle, les contributions politiques, la tenue des livres, la protection de l'environnement. Le thème de la déontologie s'intéresse davantage aux cadeaux, aux conflits d'intérêts et à la publicité trompeuse.

\section{Interdictions, tolérances et jugements}

Ces interdictions peuvent être fortes, c'est-à-dire sans équivoque, ou faibles, soit énoncées au conditionnel présent ou démontrant autrement que le comportement en question n'est pas souhaité dans l'entreprise. Elles peuvent comporter des exceptions ou être valides sauf avec autorisation préalable. Les catégories où se retrouvent les sujets traités par la majorité des entreprises (entre 11 et 27 compagnies) sont aussi celles où les éventails du nombre d'interdictions sont les plus larges (un maximum de 7 à 18 interdictions, selon les sujet traités) : conflits d'intérêts (18), concurrence déloyale (16), relations avec les employés (10), cadeaux et avantages (7), utilisation de renseignements confidentiels (7). Ce résultat nous pousse à croire que plus la source de conflits éthiques est partagée par un nombre élevé d'entreprises dans leurs codes d'éthique, plus nous pourrions y retrouver un grand nombre d'interdictions. Mais cela ne fait état que d'une tendance. Car nous retrouvons dans la catégorie dont nous parlons (entre 11 et 27 compagnies) des sujets qui contiennent très peu d'interdictions. Enfin, la grande majorité des sources de conflits éthiques $(80,7 \%)$ comportent au moins une interdiction.

Certaines sources de conflits éthiques sont sujettes à tolérance. Les conduites peuvent être tolérées purement et simplement, ou être tolérées à certaines 
conditions explicites, ou encore être tolérées avec autorisation préalable. Dans la majorité des cas, nous ne retrouvons qu'une ou deux tolérances explicitement énoncées dans le texte.

Près de la moitié $(46,1 \%)$ des sources de conflits éthiques font l'objet d'un comportement toléré. Dans le cas des comportements laissés au jugement des employés, il n'y a, en général, qu'une seule référence à ce sens du jugement dans le texte. Près du tiers $(30,8 \%)$ des sources de conflits éthiques font état du discernement éthique des destinataires du code. Plus du tiers des sujets (35\%) ne comportent que des interdictions. Très peu $(5 \%)$ ne contiennent que des tolérances ou que des interdictions et des jugements, et aucun ne comporte que des jugements. Près du quart des sujets traités comportent à la fois des interdictions, des tolérances et des jugements. Un certain nombre ne comporte que des interdictions et tolérances. Dans une proportion similaire (15\%), peu de sujets ne contiennent aucune interdiction, tolérance ou jugement.

\section{En cas de doute, qui consulter?}

En cas de doute sur l'interprétation du code, 16 codes d'éthique $(45,6 \%)$ prévoient à qui l'employé devrait s'adresser. Un seul code d'éthique $(6,2 \%)$ suggère à l'employé de s'en remettre à son jugement personnel. Un bon nombre $(37,2 \%)$ de codes suggèrent aux employés de communiquer avec les services juridiques ou le contentieux de la compagnie, ou le vice-président affaires juridiques ou le secrétaire corporatif. La majorité des codes d'éthique sont, en fait, des politiques d'entreprises? ${ }^{7}$. Ces résultats démontrent une tendance notoire à référer aux avocats en cas de problème d'éthique, car on fait l'identification entre le juridique et l'éthique (ce qui est considéré éthique, c'est ce qui est conforme aux lois). Mais, dans la majorité des cas $(62,5 \%)$, on réfère les employés à leur supérieur hiérarchique. La moitié de ces entreprises sont américaines, et dans la même proportion, il s'agit de politiques d'entreprise.

\section{En cas de violation du code d'éthique, à qui dénoncer?}

En cas de transgression du code d'éthique, 15 entreprises $(41,6 \%)$ prévoient à qui faire la plainte. Un certain nombre $(20 \%)$ suggèrent de faire suivre la plainte aux services juridiques, au contentieux, au vice-président affaires juridiques ou au secrétaire corporatif. Notez que le pourcentage de référence au secteur juridique est ici presque deux fois moins élevé que dans le cas d'une question d'interprétation du code d'éthique. Mais la majorité des entreprises $(66,7 \%)$ demandent de faire la dénonciation à leur supérieur immédiat. La grande majorité de ces codes d'éthique sont des politiques d'entreprise. Dans un seul code d'éthique, il est mentionné de dénoncer à des autorités plus élevées si son supérieur immédiat est impliqué dans l'infraction. Fait intéressant à noter, la moitié des entreprises qui favorisent la consultation du supérieur hiérarchique en cas de doute sur l'interprétation du code correspond au même pourcentage d'entreprises qui demandent à leurs employés de dénoncer des violations du code d'éthique également à leur supérieur hiérarchique. Une certaine consistance dans l'intervention pourrait être visée. Enfin, très peu de codes d'éthique $(13,3 \%)$ prévoient d'autres personnes à qui faire la dénonciation : vice-président ressources humaines, responsable de l'éthique (ethics officer).

\section{L'éthique des affaires et l'environnement législatif national}

Comme tel, l'environnement législatif national n'explique pas directement les différences identifiées dans les codes d'éthique respectifs de chaque pays considéré. Certes, si l'on envisage formellement règle par règle le cadre juridique des États-Unis, du Canada et du Québec ainsi que des pays de l'Europe continentale, tout n'est pas parfaitement symétrique. De façon générale, le droit américain apparaît plus exigeant à l'égard des entreprises ${ }^{8}$. Les lois et les règlements sont nombreux, stricts et couvrent davantage d'aspects que les autres. Cette couverture législative et réglementaire est attribuable à une culture fondée sur une grande confiance dans le pouvoir du droit d'influencer positivement et efficacement l'évolution sociale, y compris évidemment celle du monde des affaires. Le droit y est en quelque sorte sacralisé : le droit est essentiellement un bon et juste outil de régulation sociale. Et il est perçu comme jouant si bien son rôle qu'il finit par représenter la réalité sociale elle-même. On imagine plus difficilement qu'ailleurs, par exemple, que le règlement des différends se fasse autrement que par un appel direct aux règles et normes juridiques.

Au Canada et au Québec, les lois et les règlements sont très présents, eux aussi. Cependant, ils demeurent fortement dépendants, dans leur interprétation et leur 
L'environnement législatif et réglementaire qui prévaut au Canada et au Québec est façonné par l'entremise d'une approche globale, ou holistique. On conçoit que des règles juridiques précises sont nécessaires afin d'assurer un bon fonctionnement du monde des affaires; mais on conçoit également que ces règles doivent constamment être envisagées dans un contexte plus général, de façon à les placer en synergie avec l'ensemble des considérations qui guident la société.

application, de ce qui se présente sous la forme de principes généraux. C'est ainsi que l'éthique canadienne et québécoise des affaires est plus abandonnée que sa contrepartie américaine à des considérations fondamentales. Pour s'en tenir au cas du Québec, l'on pourrait avancer que le concept de bonne foi est probablement celui qui domine entre tous 9 . Il occupe une place fondamentale à l'intérieur du Code civil du Québec $^{10}$, ce qui lui permet d'agir en tant que pièce maîtresse pour l'évaluation de la justesse des comportements préconisés par les gens d'affaires. En somme, l'environnement législatif et réglementaire qui prévaut au Canada et au Québec est façonné par l'entremise d'une approche globale, ou holistique. On conçoit que des règles juridiques précises sont nécessaires afin d'assurer un bon fonctionnement du monde des affaires; mais on conçoit également que ces règles doivent constamment être envisagées dans un contexte plus général, de façon à les placer en synergie avec l'ensemble des considérations qui guident la société.

Quant à l'Europe continentale, elle se situe à mi-chemin entre les positions identifiées précédemment $^{11}$. Il tend à s'y développer une convergence juridique à partir des forces et des politiques associées à l'unification européenne. Et cette convergence juridique se traduit par une production législative et réglementaire substantielle. Toutefois, celle-ci semble vouloir s'apprécier à la lumière de normes et de principes implicites, qui logent au sein de ce qu'il convient d'appeler une conscience européenne. L'environnement législatif et réglementaire y est donc hybride : il fait appel, à la fois, à la clarté et à la précision caractéristiques du droit, et à la profondeur et à la luminosité que celui-ci est également capable de procurer. Ainsi, l'asymétrie formelle entre les systèmes juridiques mentionnés précédemment ne constitue pas le facteur déterminant dans l'explication des différences nationales existant en matière d'éthique des affaires. Parce que, de toute évidence, ces différences sont patentes. Par exemple, dans son étude intitulée "A Comparison of Business Ethics in North America and Continental Europe», George Enderle expose bien les deux caractéristiques principales de ces particularismes nationaux :

«Europeans (compared to the situation in the United States) seem to be more reluctant to speak about ethical issues in public. [...] Moreover, they often use indirect ways in order to express concern, particularly legal regulations and negotiations between social partners. [...] Also Europeans are predominantly concerned with systemic macro-issues while North American mainly deal with personal micro-issues $^{12} . »$

Si les divergences éthiques ne trouvent pas principalement leur explication dans le cadre juridique, c'est en raison du rôle significatif joué par la culture, et en particulier de la religion nationale ou majoritaire à cet égard. Le réservoir de principes de pensée et de valeurs, constitué par la culture, modèle et ajuste davantage les règles éthiques propres à chaque pays que ne le fait le simple droit. Les principaux paramètres, issus de la culture et de la religion nationale ou majoritaire, influencent directement la moralité sociale d'un pays. Ainsi, il importe de souligner le poids des forces idéologiques. Celles-ci expliquent qu'aux États-Unis, où règne la pensée pragmatiste, l'éthique des affaires soit évaluée sur les plans de la rationalité et de l'efficacité alors qu'ailleurs, elle soit plus appréciée pour sa valeur «in se» et «per se». À partir des perspectives de développement économique, il est aussi possible de faire une place plus ou moins grande aux considérations éthiques. La distribution du pouvoir au sein de la société, et particulièrement dans le monde des affaires, représente aussi un facteur d'impact additionnel. À titre d'exemple, les programmes d'implication et de participation des employés à la gestion de l'entreprise, 
comme aux États-Unis, sont reconnus pour favoriser un sens éthique accru. Les institutions académiques, groupes d'intérêt public et groupes communautaires contribuent aussi à façonner l'éthique des affaires nationale selon une orientation donnée. Ces institutions organisent des séminaires, réalisent des recherches, bref animent la pensée de telle sorte qu'elles ne sont pas étrangères à l'allure que prend l'éthique dans un pays spécifique. Finalement, il est intéressant de tenir compte du degré d'ouverture ou de fermeture de la culture nationale. Le principe veut que la moralité sociale soit enrichie de ce qu'elle intègre en provenance de l'extérieur, tout en se définissant par ce qu'elle exclut et par ses innovations. Ce principe permet d'illustrer de grandes différences culturelles entre les États-Unis, le Canada, le Québec et certains pays d'Europe occidentale comme l'Angleterre, la France et la Belgique.

Si les divergences éthiques ne trouvent pas principalement leur explication dans le cadre juridique, c'est en raison du rôle significatif joué par la culture, et en particulier de la religion nationale ou majoritaire à cet égard. Le réservoir de principes de pensée et de valeurs, constitué par la culture, modèle et ajuste davantage les règles éthiques propres à chaque pays que ne le fait le simple droit.

\section{Mise en situation de l'auto-réglementation dans les entreprises}

Comment interpréter ces résultats? Comment les comprendre à la lumière des enjeux et des problématiques qui façonnent l'époque actuelle? Et comment les envisager dans l'évolution possible ou probable que connaîtront ces enjeux et problématiques? Il s'agit ici de mesurer les possibilités et limites de développement de l'éthique de l'entreprise en regard des différents concepts contractualistes ou communautaristes de l'entreprise et de sa responsabilité sociale. Nous analyserons également la question de l'autoréglementation dans le contexte de la mondialisation des marchés.

\section{Auto-réglementation et responsabilité sociale de l'entreprise}

La conception contractualiste renvoie à une vision classique de l'entreprise, dont le but premier est de maximiser l'avoir des actionnaires. En conformité avec la poursuite de cet objectif, les contractualistes s'opposent, de façon générale, à tout type de règles qui dictent ou orientent les relations de l'entreprise. La seule responsabilité sociale que peut avoir l'entreprise consiste à maximiser ses profits. La manière dont ils croient que cette maximisation des profits résultera en un plus grand bien-être collectif, une prospérité nationale qui a des retombées pour l'ensemble des citoyens, varie selon l'un ou l'autre auteur. Il n'en demeure pas moins qu'ils croient tous en la capacité du libre-marché d'améliorer le bienêtre collectif et, dans certains cas même, de contribuer à une plus grande justice sociale ${ }^{13}$. L'optique antiréglementaire propre à la conception contractualiste laisse l'initiative aux différents intervenants qui gravitent autour de l'entreprise (stakeholders) d'établir et de définir eux-mêmes leurs rapports. La croissance de la valeur de l'entreprise, en particulier de l'avoir des actionnaires, constitue le fil conducteur de la dynamique qui procède à l'établissement et à la définition de ces rapports. En conséquence, il va de soi que l'on reconnaisse aux gestionnaires la plus grande latitude possible dans l'exécution de leur mission. Toute restriction ou spécification à cet égard est présumément contre-productive. Par ailleurs, les contractualistes ne nient pas que les «non-actionnaires» puissent subir des inconvénients, voire des préjudices, en raison de décisions prises au nom du principe d'accroissement de la richesse de l'entreprise. Cependant, ils estiment que ces «non-actionnaires» disposent du pouvoir nécessaire pour se protéger contre de tels aléas par différentes méthodes contractuelles, et qu'à défaut de l'avoir fait, ils peuvent, d'eux-mêmes, obtenir compensation auprès des instances compétentes. Les contractualistes feront ainsi une objection de principe à ce que des codes d'éthique interviennent dans l'évolution de l'entreprise. Pour eux, les personnes, autant physiques que morales, devraient être libres de faire leurs propres choix quant à la façon de conduire leur vie. De ce point de vue, les règles qui prescrivent des formes de redistribution de la richesse ou empêchent les personnes de conclure tel ou tel type de rapports sont vues comme contestables. En toutes circonstances, la pierre angulaire des conduites individuelles et collec- 
tives demeure le principe hautement individualiste du simple consentement libre et éclairé.

L'optique anti-réglementaire propre à la conception contractualiste laisse l'initiative aux différents intervenants qui gravitent autour de l'entreprise (stakeholders) d'établir et de définir eux-mêmes leurs rapports. La croissance de la valeur de l'entreprise, en particulier de l'avoir des actionnaires, constitue le fil conducteur de la dynamique qui procède à l'établissement et à la définition de ces rapports.

Par contre, les communautaristes considèrent tout à fait légitime et utile d'élaborer et d'appliquer des règles afin de structurer les relations entre les diverses composantes de l'entreprise. Cette attitude repose sur la conviction que des «règles» sont indispensables afin de contrecarrer les effets néfastes encourus par ces composantes, résultant de la volonté d'atteindre l'objectif de maximisation de l'avoir des actionnaires. Cela s'explique, notamment, par un scepticisme manifesté envers le contrat comme outil de prévention et de protection. Pourquoi? D'une part, l'information nécessaire pour prendre une décision éclairée n'est pas toujours facile à obtenir, une information qui perd, par ailleurs, rapidement de son actualité à cause des facteurs de changement et d'innovation intrinsèques à l'entreprise, et d'autre part, la coordination contractuelle des composantes de l'entreprise s'avère une tâche pratiquement utopique dans la réalité. Les communautaristes se démarquent également des contractualistes par l'attention sensible qu'ils portent aux effets sociaux découlant des actions de l'entreprise. Ils perçoivent l'entreprise comme représentant plus qu'un simple ensemble de contrats. Les entreprises sont de véritables institutions dont la conduite produit de véritables répercussions dans la société et la nature. En ce sens, les problématiques associées à l'entreprise leur apparaissent plus larges, et le contrat moins fiable en tant qu'instrument de régulation.

Même si l'approche communautariste est assez diversifiée en ce qui a trait au concept même de responsabilité sociale de l'entreprise, un fil conducteur s'en dégage : c'est la valeur d'appartenance à une communauté et les obligations de droit naturel qui en ressortent. En effet, l'appartenance à une communauté rend ses membres responsables de certaines obligations qui vont au-delà de celles pouvant exister par l'entremise du contrat. Il n'est pas possible d'ignorer ou de sous-estimer les forces et les dimensions intrinsèques à la communauté : elles créent une sorte d'héritage perpétuel qui procure une vitalité et un sens de la direction à l'interdépendance à la base des liens unissant ses membres. Le recours à des règles extracontractuelles comme des codes d'éthique mérite, pour les communautaristes, une ouverture d'esprit pleine et entière.

Les communautaristes considèrent tout à fait légitime et utile d'élaborer et d'appliquer des règles afin de structurer les relations entre les diverses composantes de l'entreprise. Cette attitude repose sur la conviction que des «règles» sont indispensables afin de contrecarrer les effets néfastes, encourus par ces composantes, résultant de la volonté d'atteindre l'objectif de maximisation de l'avoir des actionnaires.

\section{Auto-réglementation et mondialisation}

Plusieurs phénomènes sont associés à la mondialisation des marchés, sans compter les remises en question du bien-fondé de cette mondialisation en parlant par exemple d'une «mondialisation de la misère». Le phénomène culturel de mondialisation des affaires est multidimensionnel. Sans entrer dans les détails, il soulève la question de l'idolâtrie de la compétitivité ainsi que celle d'une surenchère de la moralité interne des marchés par rapport à sa moralité externe issue d'idéologies particulières ${ }^{14}$. Pour notre propos, il s'agit de se pencher sur le phénomène de complexité croissante qui est lié à la mondialisation et d'anticiper le genre d'influence qu'il peut exercer sur l'éthique de l'entreprise. Dans la mesure où l'on tient pour acquis la prémisse à l'effet que les normes de comportement sont culturellement déterminées, il s'ensuit, du fait de la mondialisation, une diversité plus grande dans les 
rapports d'affaires ainsi qu'un potentiel de différends accentué. Comment réagir, le plus justement et finement possible, face à ces données? Le développement d'un esprit de collégialité et de communication devrait entourer l'essor de l'éthique de préférence à une stratégie fondée sur l'imposition de normes strictes et précises. Ces dernières ne sont pas dénuées de toute utilité, même dans un contexte international.

Dans les rapports entre partenaires d'affaires appartenant à différentes cultures ou religions, les règles doivent être placées au centre d'un dialogue qui facilite l'ajustement mutuel entre diverses façons de voir les choses. L'éthique se profile, dès lors, sous l'angle d'une «structure de compréhension» à travers laquelle chacun peut s'enrichir au contact de l'orientation de valeurs propre à son interlocuteur ou à son partenaire d'affaires. En prospective, la mondialisation semble tracer les axes suivants de mûrissement potentiel pour l'éthique de l'entreprise dans les affaires internationales. Une approche interculturelle ou interreligieuse dans les affaires internationales supposerait de : 1) pour chaque partenaire d'affaires, mieux comprendre les enracinements culturels et religieux des normes éthiques de comportement en affaires; 2) pour chaque partenaire d'affaires, s'assurer de mieux comprendre les enracinements culturels et religieux des normes éthiques qui s'appliquent à l'autre, et afin qu'un enrichissement mutuel puisse naître, dans le dialogue entre les partenaires d'affaires sur les questions éthiques dans les affaires internationales; 3) procurer une cohérence minimale sans laquelle les significations en découlant ne pourraient pas être partagées par les intéressés; 4) être compatible avec le dynamisme social et les mutations qu'il engendre; 5) demeurer en perpétuel ajustement au monde extérieur, c'est-à-dire ouvert, placé en position d'interaction tout en respectant les caractéristiques culturelles et religieuses de ses partenaires d'affaires qui viennent influencer la moralité externe des marchés ${ }^{15}$.

\section{Un regard pluriel sur l'éthique des affaires}

La nature et le sens de l'éthique ne participent pas de l'observation de faits qui sont extérieurs à la pensée humaine, et constituant un cadre de référence neutre, objectif et constant. Nature et sens découlent plutôt d'une réalité interne, une vision qu'Amselek exprime,
Dans les rapports entre partenaires d'affaires appartenant à différentes cultures ou religions, les règles doivent être placées au centre d'un dialogue qui facilite l'ajustement mutuel entre diverses façons de voir les choses. L'éthique se profile, dès lors, sous l'angle d'une «structure de compréhension» à travers laquelle chacun peut s'enrichir au contact de l'orientation de valeurs propre à son interlocuteur ou à son partenaire d'affaires.

dans la sphère juridique, par la formule «le droit dans les esprits». Dès lors, les frontières de l'éthique débordent le cadre positiviste constitué, par exemple, des seuls codes d'éthique. Ce qu'est l'éthique devient lié à un contexte de vie humaine rempli d'attitudes, d'expériences et d'espoirs. De même, cette réalité interne fait-elle de l'éthique une entité soluble : les données éthiques se meuvent au travers d'autres types de données (juridiques, économiques, politiques, sociales, etc.), créant ainsi des zones de contact et d'influence réciproque dont les résultantes sont plurielles et souvent indéterminées. L'équation éthique - développement est, par conséquent, abordée sous l'angle de la complexité. Vient compléter ce dernier aspect celui de l'interaction. Un complément qui traduit une double préoccupation : une première pour une compréhension de la façon avec laquelle un ou des éléments de la relation éthique - développement se diffusent et se transforment d'un site humain à un autre, et une seconde, pour une saisie de ce qu'un site humain a pu faire (innover, consolider, etc.) d'un ou de tels éléments. Finalement, ces énoncés s'allient à une approche qui voit dans le rapport éthique - développement un creuset à démêler par interprétation. Le souci d'établir des liens sûrs avec des répercussions prévisibles laisse place ici à une volonté de mettre en lumière le tissu de significations qui relie éthique et développement.

En vertu de la conception de l'opulence comme principe clé de toute société matérialiste, éthique et développement consistent essentiellement en un processus de croissance économique. Cela se traduit 
par la prise en compte de facteurs et d'indicateurs tels la montée de la production, l'essor de la productivité et l'augmentation du revenu par habitant. Par contraste, et afin de rompre avec l'idée que nous sommes dans le siècle de l'argent, la pensée émergente vise à replacer les valeurs dans une perspective d'éléments intrinsèquement estimés et valorisés. Les valeurs étant délestées de leur rôle purement utilitaire, le lien éthique - développement se rapproche davantage d'un processus «qui accroît la liberté effective de ceux qui en bénéficient de poursuivre toute activité à laquelle ils ont des raisons d'attacher de la valeur».

La nature et le sens de l'éthique ne participent pas de l'observation de faits qui sont extérieurs à la pensée humaine, et constituant un cadre de référence neutre, objectif et constant. Les frontières de l'éthique débordent le cadre positiviste constitué, par exemple, des seuls codes d'éthique. Ce qu'est l'éthique devient lié à un contexte de vie humaine rempli d'attitudes, d'expériences et d'espoirs.

L'éthique des affaires est appelée à contribuer à la recherche d'un modus vivendi capable de satisfaire un besoin inné de raison d'être au monde. Plus spécifiquement, l'éthique d'une entreprise repose sur une pierre angulaire à deux faces : l'une qui concourt à son sain épanouissement, et l'autre qui assure une solidarité avec son entourage. La première facette réfère à l'étendue des possibilités qui s'offrent à une entreprise de se réaliser, c'est-à-dire de choisir et de satisfaire ses aspirations, ses buts organisationnels. Ainsi, l'existence d'une entreprise sera perçue comme constituée d'une série d'actions, de gestes ou de projets et de visions qui s'intègrent dans un ensemble de réalisations. La seconde facette, la solidarité, vise à imprégner les diverses activités valorisées aux fins de l'épanouissement d'un sentiment de responsabilité et de communauté d'intérêts. Elle implique que la vitalité du modus vivendi évoqué précédemment exige davantage que le seul appel à l'épanouissement. Il faut aussi que celui-ci se fasse dans le respect de ce que Ricardo Petrella appelle les "principes d'existence de l'autre et de coexistence ${ }^{16 » !}$ D'après Petrella ${ }^{17}$, ces principes sont à la base d'un bien commun matériel et immatériel qu'il place au coeur d'un contrat social mondial. Dans la mesure où vivre ensemble possède un sens en soi, c'est-à-dire qui va au-delà de la simple somme des buts organisationnels de l'entreprise, la solidarité apparaît comme un complément nécessaire à la réalisation de ces buts.

L'éthique d'une entreprise repose sur une pierre angulaire à deux faces : l'une qui concourt à son sain épanouissement, et l'autre qui assure une solidarité avec son entourage. La première facette réfère à l'étendue des possibilités qui s'offrent à une entreprise de se réaliser, c'est-à-dire de choisir et de satisfaire ses aspirations, ses buts organisationnels. La seconde facette, la solidarité, vise à imprégner les diverses activités valorisées aux fins de l'épanouissement d'un sentiment de responsabilité et de communauté d'intérêts.

L'éthique des affaires ${ }^{18}$ peut se frayer avantageusement un chemin dans le courant de pensée dont nous venons de faire état. Il reste cependant qu'afin de mesurer pleinement ses possibilités et limites, il importe de l'analyser en fonction de deux conceptions différentes de l'entreprise, la conception contractualiste et la conception communautariste. Selon que l'une ou l'autre est adoptée, il s'avère plus aisé de comprendre la portée relative d'un code d'éthique dans une entreprise donnée, de même que l'apport effectif susceptible d'être fourni par les entreprises au débat sur la redéfinition du juste et du bien.

\section{Notes et références}

1 Michel Dion est vice-doyen à l'enseignement à la Faculté de théologie, d'éthique et de philosophie de l'Université de Sherbrooke.

2 Louis Marquis est doyen de la Faculté de droit de l'Université de Sherbrooke.

3 RAIBORN, Cecily and Dinah PAYNE (1990). «Corporate Codes of Conduct: A Collective Conscience and Continuum», Journal of Business Ethics, vol. 9, p. 881.

4 US DEPARTMENT OF LABOR (1996). The Apparel Industry and Codes of Conduct: A Solution to the 
International Child Labor Problem, p. 46 ; COMPA, L. and T. HINCHLIFFE-DARRICARRÈRE (1995). «Enforcing International Labor Rights Through Corporate Codes of Conduct», Columbia Journal of Transnational Law, vol. 33, p. 686.

DION, M. et R. LESCARBEAU (1995). «Les codes d'éthique des entreprises manufacturières au Québec : état des perceptions des dirigeants », Ethica, vol. 7, $\mathrm{n}^{\circ} 1$, p. 113-144 ; LEFEBVRE, M. and J. B. SINGH (1992). «The Content and Focus of Canadian Corporate Codes of Ethics », Journal of Business Ethics, vol. 11, p. 799-808; DION, Michel (2000). «Valeurs et croyances organisationnelles dans les codes d'éthique d'entreprises manufacturières au Québec », Revue organisations et territoires, vol. 9, $\mathrm{n}^{\circ} 1$, hiver, p. 47-54.

Le thème de la responsabilité sociale de l'entreprise est très complexe. Ce que nous avons noté ici,c'est uniquement là où il est explicitement nommé tel quel. D'ailleurs, le concept même de responsabilité sociale adopté par une entreprise n'est pas nécessairement le même dans ses documents les plus représentatifs. Ainsi, le code d'éthique peut véhiculer un concept beaucoup plus large que celui qui sera reflété dans le rapport annuel de l'entre prise. À cet effet, voir M. DION (2001). «Le développement réel de l'éthique de l'entreprise au Québec », Déréglementation et éthique d'en treprise ; M. DION (2001). «La responsabilité sociale de l'entreprise : entre les Murailles de Chine et la boîte de Pandore », L'État, la société civile et l'économie. Turbulences et transformations en période de décroissance, G. Giroux (éd.), Sainte-Foy, PUL - L'Harmattan, p. 145-173.

7 Nous reprenons ici la division tripartite définie par DION (1994) : philosophie de gestion (définition des principales valeurs organisationnelles), politique d'entreprise (définition des normes de comportement éthique dans l'entreprise, sans aucune définition des valeurs organisationnelles principales), et code de conduite (définition des valeurs organisationnelles et normes de comportement dans l'entreprise, lignes directrices, procédure de révision et de renforcement du code). Selon l'enquête provinciale menée par Dion et Lescarbeau en 1993, 11,1 \% des entreprises manufacturières ont une philosophie de gestion, 46,3\% ont une politique d'entreprise et $42,8 \%$ ont un code de conduite (M. DION [1994]. L'éthique de l'entreprise, Montréal, Fides, p. 189-190).

FOLSOM, R. et A. LEVASSEUR (1994). Pratique du droit des affaires aux États-Unis, Paris,Dalloz; STONE, F. (1965). Institutions fondamentales du droit des États-Unis, Paris, LGDJ.
9 LEFEBVRE, B. (1995-1996). La bonne foi : notion protéi forme, RDUS, $\mathrm{n}^{\mathrm{O}} 26$, p. 321.

10 Voir les articles 6 et 7.

11 SHAPIRA, J. (1986). Le droit européen des affaires, Paris, PUF ; BRUNETEAU, B. (1996). Histoire de l'unification européenne, Paris, Armand Colin.

12 ENDERLE, Georges (1996). «A Comparison of Business Ethics in North America and Continental Europe », Business Ethics. A European Review, p. 33-46.

13 SMITH, Adam J. (1976). Recherche sur la nature et les causes de la richesse des nations. Les grands thèmes, Paris, NRF, Gallimard, (Collection Idées) ; HAYEK, Friedrich A. (1973-1976-1979). Law, Legislation and Liberty, Chicago, The University of Chicago Press, 3 vol. ; FRIEDMAN, Milton (1962). Capitalism and Freedom, Chicago, The University of Chicago Press.

14 BUONO, Anthony F. and Larry NICHOLS (1985). Corporate Policy, Values and Social Responsibility, NewYork, Praeger ; BAUER, Raymond A., L. Terry CAUTHORN and Ranne P.WARNER (1978). "The Management Process Audit Manual», Research in Corporate Social Performance and Policy, vol. 1, p. 265-277 ; CARROLL, Archie B. (1977). Managing Corporate Social Responsibility, Boston, Little, Brown.

15 SETHI, S. Prakash (1979). «A Conceptual Framework for Environmental Analysis of Social Issues and Evaluation of Business Response Patterns», Academy of Management Review, january, p. 65 ff.

16 McCOY, Charles S. (1985). Management of Values. The Ethical Difference in Corporate Policy and Performance, New York, Harper and Row ; FREEMAN, R. Edward (1984). Strategic Management: A Stakeholder Approach, Marshfield, Pitman.

17 PETRELLA, Ricardo (1997). Écueils de la mondialisation : urgence d'un nouveau contrat social, Saint-Laurent, Fides.

18 Voir DION, Michel (2001). «La responsabilité sociale de l'entreprise : entre les Murailles de Chine et la boîte de Pandore», L'État, la société civile et l'économie ; DION, Michel (1998). Investissements éthiques et régie d'entreprise. Entre la mondialisation et la mythologie, Montréal, $\mathrm{n}^{\circ}$ 11, Médiaspaul. (Collection Interpellations); DION, Michel (1997-1998). «A Typology of ValueOrientations as Applied in the Business World», Journal of Oriental and African Studies (Greece), vol. 9, p. 95-110. 